\title{
Management of Iatrogenic Duodenal Perforation with Bilateral Pneumotho- rax Following ERCP; A Case Report and Review of the Literature
}

\author{
Christophoros S Kosmidis, Georgios D Koimtzis*, Stefanos K Atmatzidis, Ioannis Koskinas, Stylianos V Mantalovas, Vyron \\ Alexandrou, Nikolaos Varsamis, Eleni Georgakoudi, Konstantinos Sapalidis and Isaac I Kesisoglou
}

$3^{\text {rd }}$ Department of surgery, University Hospital of Thessaloniki AHEPA, Aristotle's University of Thessaloniki, Thessaloniki, Greece

"Corresponding author: Georgios D Koimtzis, 3rd Department of surgery, University Hospital of Thessaloniki AHEPA, Aristotle's University of Thessaloniki, Thessaloniki, Greece. Tel: +30-6971879241; Email: drgxkoimtzis@gmail.com

Citation: Kosmidis CS, Koimtzis GD, Atmatzidis SK, Koskinas I, Mantalovas SV, et al. (2019) Management of Iatrogenic Duodenal Perforation with Bilateral Pneumothorax Following ERCP; A Case Report and Review of the Literature. J Surg 13: 1243. DOI: $10.29011 / 2575-9760.001243$

Received Date: 27 July, 2019; Accepted Date: 02 August, 2019; Published Date: 06 August, 2019

\begin{abstract}
Introduction: Endoscopic-Retrograde-Cholangiopancreatography (ERCP) is one of the most commonly used procedures for the diagnosis and treatment of hepatobiliary and pancreatic diseases. Although it is considered a relatively safe procedure, ERCPinduced duodenal perforation is a rare but severe complication with an incidence of approximately $1 \%$ and a mortality rate that reaches up to 36\%, with most perforations being related to Endoscopic Sphincterectomy (EST) and catheterization of the ampulla. Surgical management is considered the treatment of choice in these cases, despite the fact that there have been recent studies, which suggest that conservative management can be successful in a carefully selected group of patients.

Case Report: We present a case of a 78-year-old female patient who suffered from symptomatic cholelithiasis and choledocholethiasis with an episode of acute pancreatitis in the past. The patient was submitted to ERCP and EST. During sphincterotomy, a perforation of the duodenum was suspected, therefore shortly after a Computed Tomography scan (CT) was performed, which revealed the presence of a massive amount of air in the retroperitoneal space accompanied by bilateral pneumothorax. A thoracic tube was immediately placed in the right hemithorax, where the amount of air was much greater and an exploratory laparotomy was performed, which confirmed the diagnosis and revealed the presence of air bubbles in the paraduodenal area and the right paracolic groove without bile leakage. Drainage was placed in the paraduodenal space. Additionally, a cholecystectomy was performed. The postoperative course was uncomplicated and the patient was discharged on the 11th postoperative day.

Conclusions: Retroperitoneal duodenal perforation is an uncommon, but potentially fatal complication following ERCP. Early diagnosis requires a high degree of clinical suspicion and is crucial for a positive outcome. Although conservative management has been suggested in a selected category of patients, surgery seems to remain the treatment of choice, but large-scale case series are still missing to achieve consensual guidelines.
\end{abstract}

Keywords: Complication; Endoscopic Retrograde Cholangiopancreatography (ERCP); Duodenal perforation

\section{Introduction}

ERCP is nowadays the procedure of choice for diagnostic and curative purposes in many biliary and pancreatic diseases [1] Despite the wide application of the procedure, the complication rate still remains relatively high $(15.9 \%)$ and is related with an overall mortality rate of $1 \%$ [2]. The most severe and potentially fatal complication is duodenal perforation with an incidence rate of $0.14-1.3 \%$ and a mortality rate of $4.2-29.6 \%[1,3]$. However, recently, lower mortality rates and better outcomes have been reported, being attributed to early detection and selection of the appropriate treatment $[1,4]$. Duodenal perforation usually presents with clinical symptoms of abdominal pain and vomiting, imitating the most common ERCP-induced complication, which is acute pancreatitis [5]. Changes in respiratory and heart rate, decreasing oxygen saturation, subcutaneous emphysema and dyspnea indicate pneumomediastinum and/ or pneumothorax, which are highly suggestive of duodenal perforation [6]. Therefore, a high level of early clinical suspicion is important, whereas CT scan is the most 
useful imaging tool to confirm the diagnosis [5]. However, the best outcomes have been reported in cases where the perforation was detected intraoperatively, although immediate detection rate ranges widely from $36 \%$ to $100 \%$ [3]. We present a case of a 78 year-old female patient with a retroperitoneal duodenal perforation accompanied by bilateral pneumothorax following an ERCP for the treatment of symptomatic choledocholithasis. Due to the large accumulation of air in the retroperitoneal space and the presence of pneumothorax, the patient was managed surgically and had an uneventful post-operative course.

\section{Case Presentation}

A 78-year-old female patient was admitted to our gastroenterology department in order to be subjected to ERCP, due to symptomatic cholelithiasis and choledocholithasis, which was suspected due to an episode of acute mild pancreatitis and confirmed afterwards by a Magnetic Resonance Cholangiopancreatography (MRCP). The rest of her medical history was free and she was on no medication. On admission, the patient's vital signs were normal with an arterial blood pressure of 130/70 $\mathrm{mmHg}$, a heart rate of 75 bpm, a temperature of $36.9 \mathrm{C}$ and a respiratory rate of 12 cycles per minute. On clinical examination no abdominal tenderness or palpable masses were found. Her laboratory test values were all within normal range, with a white blood cells count of 7,580 K/ $\mu \mathrm{L}$, a hematocrit of $41.1 \%$, a hemoglobin value of $14 \mathrm{~g} / \mathrm{dL}$, a platelet count of $188 \mathrm{~K} / \mu \mathrm{L}$, a urea value of $39 \mathrm{mg} / \mathrm{dL}$, a creatinine value of $0.66 \mathrm{mg} / \mathrm{dL}$, a $\mathrm{Na}^{+}$value of $137 \mathrm{mmol} / \mathrm{L}$, a $\mathrm{K}+$ value of 3.7 $\mathrm{mmol} / \mathrm{L}$, an INR value of 0.96 , a total bilirubin value of $0,54 \mathrm{mg} /$ $\mathrm{dL}$ and a direct bilirubin value of $0,14 \mathrm{mg} / \mathrm{dL}$. The $\gamma$-GT value was $9 \mathrm{U} / \mathrm{I}$ and ALP value was $54 \mathrm{U} / \mathrm{I}$.

During the ERCP, catheterization of the ampulla of Vater and EST were extremely difficult, due to the presence of extensive edema in this area, and the gastroenterologist was unable to remove a small gallstone that was seen in the cholangiography. A perivaterian guide wire perforation of the duodenum was suspected intraoperatively, therefore a stent placement in the common bile duct was impossible. The patient was sent back to her room and was set under surveillance of her clinical signs, in an attempt to manage her conservatively. In the next 3 hours, the patient presented with severe gradually increasing diffuse abdominal pain, thus a CT scan was scheduled immediately, which revealed the presence of a massive amount of air in the right paracolic groove, the paraduodenal space and perirenal space (Figure 1). Moreover, a bilateral pneumothorax was detected (Figure 2), more extensively at the right side, which was immediately treated with the placement of a thoracic tube in the ipsilateral hemithorax.

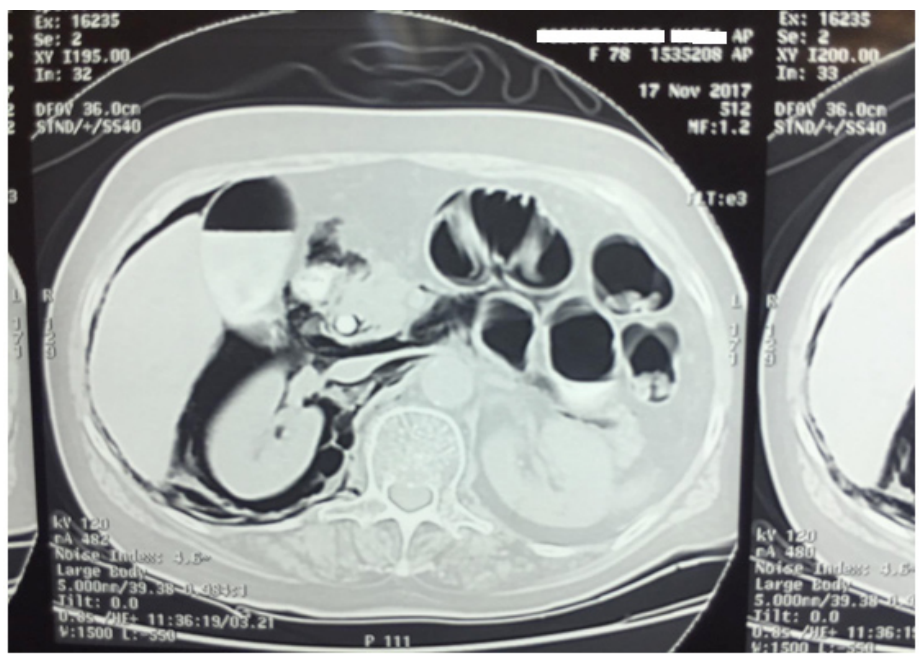

Figure 1: CT image revealing the increasing amount of air in the retroperitoneal space.

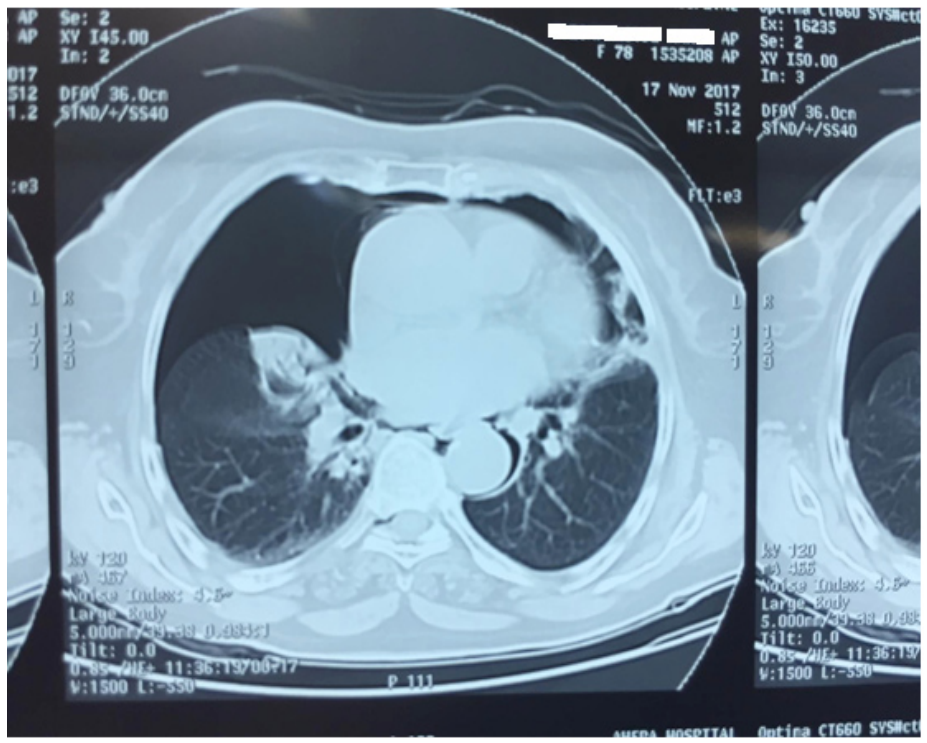

Figure 2: Ct image revealing the presence of bilateral pneumothorax, mainly in the right side.

Due to the excessive amount of air in the retroperitoneal space and the severity of the patient's clinical symptoms, the decision was made to perform an exploratory laparotomy through a midline incision in order to identify the exact location and extent of the damage. The laparotomy confirmed the radiological findings (Figure 3). In order to access the retroperitoneal space, a dissection across the line of Todd and a limited Kocher maneuver 
were performed. The perforation site could not be identified, but as there were no signs of bile leakage and an extensive damage, a further exploration of the area was not considered necessary. A drainage tube was placed across the sub hepatic area to the paraduodenal space (Figure 4). In addition, a cholecystectomy was performed for a definite treatment of cholelithiasis, whereas a choledochotomy under the present circumstances was considered unnecessary because of the small size of the gallstone left in the common bile duct and the former EST. The patient was transferred postoperatively in the intensive care unit, where she remained for one day. The postoperative course of the patient in the surgical department was uneventful. Oral intake started on the fourth postoperative day, the thoracic tube was removed on the fifth postoperative day and the abdominal drainage tube on the tenth postoperative day. The patient was discharged one day later.

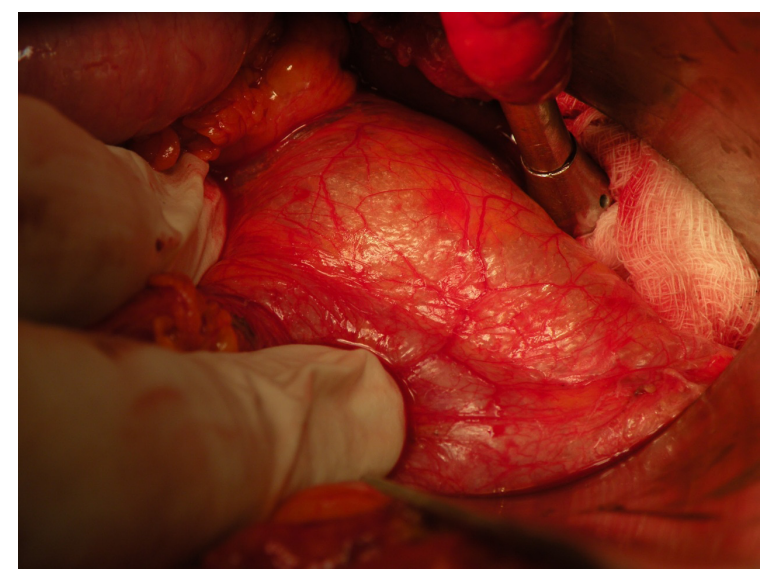

Figure 3: Presence of air bubbles in the paraduodenal space without signs of bile leakage.

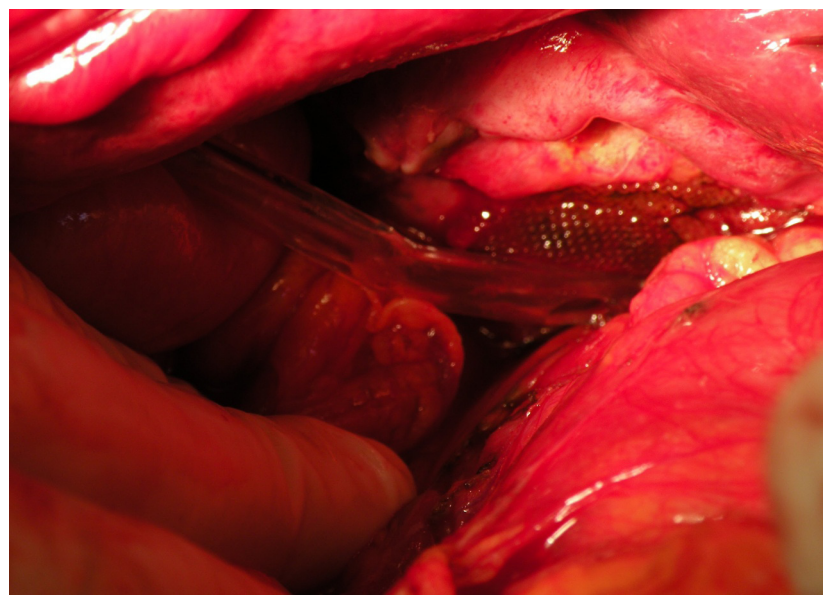

Figure 4. Drainage placed in the paraduodenal space after performong Kocher maneuver

3

J Surg, an open access journal

ISSN: $2575-9760$

\section{Discussion}

Endoscopic Retrograde Cholangiopancreatography (ERCP) is a procedure which has been widely used since its introduction in the 1970s for the diagnosis, evaluation and treatment of many hepatobiliary and pancreatic diseases [7]. However, it carries the highest complications rate among all of the endoscopic gastrointestinal procedures, such as gastroscopy and colonoscopy [7]. These complications include cholangitis, cholecystitis, pancreatitis and EST induced bleeding and duodenal perforation, which is the rarest but one of the most fatal complications, with a median incidence rate of $0.6 \%$ and mortality rate of $9.9 \%[7,8]$. Two classifications of ERCP-related perforations have been proposed in the literature [8]. The first one published in 1999 by Howard et al divided perforations into 3 types: type I includes guidewire perforations, type II periampullary perforations and type III duodenal perforations remote from the papilla [8]. The second and most widely used classification is the one proposed by Stapfer, et al. in 2000 [9]. According to this classification, there are 4 types of perforations: type I consists of lateral or medial wall duodenal perforations, requiring immediate surgical treatment, type II are peri-Vaterian injuries, which are less severe and not always require surgery, type III are distal bile duct injuries, which occur due to the usage of guidewire-basket instrumentation and type IV consists of injuries presenting with retroperitoneal air, being managed almost always conservatively $[8,9]$. Considering the Stapfer classification, our case was a type IV duodenal perforation, which was managed surgically, due to the presence of an excessive amount of air in the abdominal cavity, the concurrent presence of bilateral pneumothorax and the clinical signs of the patient.

In a study performed by Hao, et al. in 2006, among 30 post-ERCP perforations, 11 of them were periampullary, 3 were duodenal, one was esophageal, one was located on an afferent limb of a Billroth II anastomosis and in 7 patients the location of the perforation was not identified [10]. According to this study, all of the known duodenal perforations required surgery, but from the seven patients with unknown location of the perforation, 5 were managed conservatively with success. In another multicenter retrospective study by Jin et al published in 2013, 59 patients were diagnosed with ERCP-related duodenal perforation [11]. Of these patients, 34 received antibiotics combined with therapeutic fasting and hydration, 7 were subjected to endoscopic clipping immediately after injury and 18 underwent surgery [11]. Surgical treatment was selected for patients with persistent signs of peritoneal irrigation or systemic inflammation, as was in our case [11]. Three of these patients were initially managed conservatively but underwent salvage surgical treatment because of failure of the medical management [11]. Stapfer's classification was not used as an indication for conservative or surgical treatment [11]. The median time to surgery after injury detection was 4 hours (1-27) 


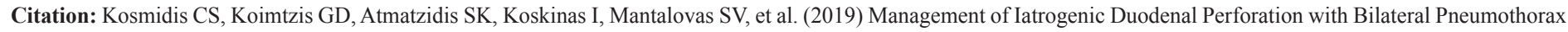
Following ERCP; A Case Report and Review of the Literature. J Surg 13: 1243. DOI: 10.29011/2575-9760.001243

and the mean length of hospitalization was 19.2 days [12]. In our case the decision for surgical treatment was based on the clinical findings of the patient and the radiological findings after the ERCP, whereas the patient was discharged on the 11th post-operative day [11]. However, in a study of 27 perforations among 1638 ERCP procedures performed by Miller et al, all type IV perforations were managed conservatively due to the lack of severe symptoms [12].

The type of surgical intervention required is chosen according to the extent of perforation induced during ERCP [13]. After the initial incision and entry in the abdominal cavity, the surgeon is obliged to choose the type of operation he will perform to the patient according to the intraoperative findings and the extent of the perforation and its complications [13]. The procedure alone may vary from a simple primary repair, where only suturing or stapling is required, to complete diversion of the enteric stream with a more sophisticated technique in addition to suturing the perforation, which will be performed if the degree of injury to the duodenum is excessive and the duodenal wall is thickened and inflamed by the perforation. The first one is preferred for more recent injuries and well vascularized and easily mobilized duodenum [13]. In these cases, the surgeon may perform a gastrojejunostomy and pyloric exclusion, in order to prevent the gastric juice from entering the damaged duodenum and divert it directly to the jejunum [13]. Prior to closure, a drainage tube is placed in the abdominal cavity near the repair site [13].

In a retrospective study performed by Preetha, et al. 18 cases of ERCP-related perforations were reported among 4030 cases. 5 of them were diagnosed intraoperatively, 4 of them were diagnosed by abdominal x-rays and the last 9 by computed tomography [14]. Eventually, all patients required surgery, with 8 of them being operated within 24 hours and the remaining 10 after the first 24 hours [14]. Based on the intraoperative findings, the procedures that were performed included duodenal diversion in the form of pyloric exclusion and gastrojejunostomy, primary duodenal repair or combined duodenal repair with T-tube diversion of the biliary tree and pyloric exclusion and gastrojejunostomy [14]. In this study, 3 deaths were reported, all of them regarding patients over 70 years old [14]. The type of injury according to Stapfer classification and the type of operation did not affect the post-operative outcome. ERCP-related pneumothorax is a very rare complication with less than 40 cases reported in the English literature [15]. The main risk factors are EST and potentially the presence of juxtapapillary diverticula [16]. The pathophysiology of this complication has not been fully understood yet $[15,17]$. One possible mechanism suggests that air from the retroperitoneum passes directly to the mediastinum and then to the pleural cavity through a rupture in the parietal pleura, while a second one suggests that congenital defects in the diaphragm allow air to pass directly in the pleural space [17]. Pneumothorax is usually bilateral $(51.35 \%)$ as it was in our case and is most commonly accompanied by pneumomediastinum and subcutaneous emphysema [15]. This condition is usually managed conservatively with or without chest tube placement [15]. However, due to the scarceness of this particular complication, there is still ongoing debate on whether the sole presence of pneumothorax is a hard indication for surgical management [17]. Nonetheless, almost $25 \%$ of the cases will eventually require surgery [15].

Finally, in a major meta analysis performed by Machado $\mathrm{N}$. in 2011, 251 cases of duodenal perforations were reported out of 10 major studies [18]. The most common cause of perforation was sphincterotomy $(25.9 \%)$, as it was in our case [18]. The predominal location of the perforation was the duodenal wall (34.5\%), perivaterian perforations came second (31.3\%), 23\% were perforations of the common bile duct and in $7.9 \%$ of the cases, the location was not identified, as in our case [18]. The diagnosis of the perforation was made within 24 hours in $78.5 \%$ of the cases with $55.8 \%$ being diagnosed during or immediately after the procedure [18]. According to this meta analysis, the most useful diagnostic tool for perforations not detected during ERCP was the CT scan [15]. This study also reveals that $62.2 \%$ of the cases were managed conservatively with a success rate of $92.9 \%$ [18]. Only 1 death was noted in a patient who also had pneumothorax, as it happened in our case [18]. The most common surgical intervention that was employed was primary duodenal repair (49\%), with or without other procedures while retroperitoneal drainage was reported in $39 \%$ of the cases [18]. Due to the inability of locating the perforation site intraoperatively, retroperitoneal drainage was the operation we opted for in our case.

\section{Conclusion}

ERCP-related duodenal perforation is an uncommon complication presenting a major diagnostic and therapeutic challenge, requiring a high degree of clinical suspicion and experience. Intraoperative or early postoperative detection is the key to a better outcome while the patient's clinical condition and the endoscopic and radiologic findings play a fundamental role in deciding the appropriate treatment, which can be either conservative or surgical.

\section{References}

1. Li Q, Ji J, Wang F, Ge X, Nie J, et al. (2015) ERCP-induced duodenal perforation successfully treated with endoscopic purse-string suture: a case report. Oncotarget 6: 17847-17850.

2. Rabie ME, Mir N, Skaini MA, I El Hakeem, A Hadad, et al. (2013) Operative and non-operative management of endoscopic retrograde cholangiopancreatography-associated duodenal injuries. Annals of The Royal College of Surgeons of England 95: 285-290. 


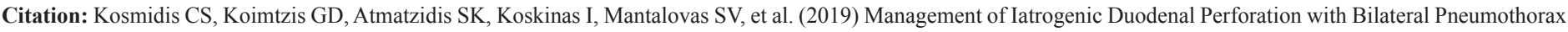
Following ERCP; A Case Report and Review of the Literature. J Surg 13: 1243. DOI: 10.29011/2575-9760.001243

3. Motomura Y, Akahoshi K, Gibo J, Kanayama K, Fukuda S, et al. (2014) Immediate detection of endoscopic retrograde cholangiopancreatography-related periampullary perforation: Fluoroscopy or endoscopy? World Journal of Gastroenterology 20: 15797-15804.

4. Park SM (2016) Recent Advanced Endoscopic Management of Endoscopic Retrograde Cholangiopancreatography Related Duodenal Perforations. Clin Endosc 49: 376-382.

5. Harvey JP (2015) Pneumopericardium, pneumomediastinum, pneumoretroperitoneum and surgical emphysema secondary to a duodenal perforation post-endoscopic retrograde cholangiopancreatography. BMJ Case Reports 2015

6. Rana M, Hussain M, Iqbal S, Ramadan O, Ashmawi S, et al. (2014) Fatal Pneumothorax Secondary to Duodenal Perforation after Endoscopic Retrograde Cholangiopancreatography. Journal of Medical Cases 5: 208-211.

7. Zhu K, Wang JP, Su JG (2017) Prophylactic ulinastatin administration for preventing post-endoscopic retrograde cholangiopancreatography pancreatitis: A meta-analysis. Experimental and Therapeutic Medicine 14: 3036-3056

8. Vezakis A, Fragulidis G, Polydorou A (2015) Endoscopic retrograde cholangiopancreatography-related perforations: Diagnosis and management. World Journal of Gastrointestinal Endoscopy 7: 1135-1141.

9. Stapfer M, Selby RR, Stain SC, Katkhouda N, Parekh D, et al. (2000) Management of Duodenal Perforation After Endoscopic Retrograde Cholangiopancreatography and Sphincterotomy. Annals of Surgery 232: 191-198.

10. Wu HM, Dixon E, May GR, Sutherland FR (2006) Management of perforation after endoscopic retrograde cholangiopancreatography (ERCP): a population-based review. HPB: The Official Journal of the International Hepato Pancreato Biliary Association 8: 393-399.
11. Jin YJ, Jeong S, Kim JH, Hwang JC, Yoo BM , et al. (2013) Clinical course and proposed treatment strategy for ERCP-related duodenal perforation: a multicenter analysis 45: 806-812.

12. Miller R, Zbar A, Klein Y, Buyeviz V, Melzer E, et al. (2013) Perforations following endoscopic retrograde cholangiopancreatography: a single institution experience and surgical recommendations 206: 180-186.

13. Dixon P, Kowdley GC, Cunningham SC (2016) The role of surgery in the treatment of endoscopic complications.Best Pract Res Clin Gastroenterol 30: 841-851.

14. Preetha M, Chung YF, Chan WH, et al. (2003) Surgical management of endoscopic retrograde cholangiopancreatography-related perforations. ANZ J Surg 73: 1011-1014.

15. Jha AK, Jha P, Priyadarshi RN, Jha SK, Purkayastha S, et al. (2018) Post-endoscopic retrograde cholangiopancreatography pneumothorax: Report of two cases and literature review. JGH Open 2: 329-332.

16. Schepers NJ, van Buuren HR (2012) Pneumothorax following ERCP: report of four cases and review of the literature. Digestive diseases and sciences 57: 1990-1995.

17. Rappaport DE, Solano JJ, Edlow JA (2017) Bilateral Pneumothoraces as a Complication of Endoscopic Retrograde Cholangiopancreatography. The Journal of Emergency Medicine 52: 573-575.

18. Machado NO (2012) Management of duodenal perforation post-endoscopic retrograde cholangiopancreatography. When and whom to operate and what factors determine the outcome? A review article 13: 18-25. 